\title{
A NOTE ON RAYS AT THE IDENTITY OPERATOR
}

\section{ROBERT SINE ${ }^{1}$}

Let $B(\mathfrak{X})$ be the algebra of all bounded linear automorphisms of a Banach space $\mathfrak{X}$ and let $S$ be the unit ball of $B(\mathfrak{X})$. If $I$ is the identity operator and $A \neq 0$, the ray generated by $A$ (through $I$ ) is the set $\{I+t A: t \geqq 0\}$. This ray is said to be tangent to $S$ at $I$ if

$$
\lim _{t \rightarrow 0_{+}} t^{-1}(\|I+t A\|-1)=0 .
$$

Bohenblust and Karlin [1] raised the question of existence of rays tangent to $S$ at $I$ from the radical. Lumer and Phillips [3] settled the conjecture in the negative by constructing a quasi-nilpotent operator on Hilbert space for which $(*)$ obtains. For this purpose the negative of the standard Volterra opreator on the $L_{2}$ space of the interval $[0,1]$ will serve as well as their example. By a modification of their example we give an example of a ray from the radical which is not only tangent to $S$ at $I$ but is actually in contact with the surface of the ball on an interval of the ray.

Let $N$ be any nonzero quasi-nilpotent in $B(\mathfrak{X})$ which satisfies $(*)$. Any operator $A$ which satisfies $(*)$ is dissipative and so generates a semigroup of contractions; $\left\|e^{t A}\right\| \leqq 1$ for $t \geqq 0$. If we set $Q=e^{N}-I$ we see that $Q$ is an analytic function of $N$ so is itself a qausi-nilpotent by the spectral mapping theorem. Now $\|I+1 Q\|=\left\|e^{N}\right\|=1$ so the ray from $Q$ is in contact with $S$ for $t=0$ and $t=1$. From the convexity of $S$ it follows that $\|\mathrm{I}+t Q\| \leqq 1$ for $0 \leqq t \leqq 1$ and from the fact that the norm must exceed the spectral radius $\|I+t Q\| \geqq 1$. Thus for $0 \leqq t \leqq 1$ the ray is on the surface of $S$.

We have thus far begged the question that $Q \neq 0$. For the Volterra operator $N$ on $L_{2}[0,1]$ we have $\|N\|=1$ and it follows at once from examination of the exponential series that $e^{-N}=I$ is not possible.

If $N$ is an arbitrary quasi-nilpotent on a Hilbert space with $e^{N}=I$ it follows at once that $e^{t N}$ is a bounded group so that Nagy's theorem that a bounded representation of the integers is similar to a unitary representation applies. The spectral mapping theorem then finishes this case.

For the setting of an arbitrary Banach space we can argue as follows. Let $T=e^{t_{0} N}$. If $e^{N}=I$ then we have a uniform bound $\left\|T^{n}\right\| \leqq B$

Received by the editors November 18, 1968.

${ }^{1}$ Supported in part by the National Science Foundation. 
for $-\infty<n<\infty$. Again from the spectral mapping theorem we have $\sigma(T)=\{1\}$ so that $T$ is a quasi-nilpotent perturbation of the identity. Then by a classical result of Gel'fand's [2, p. 128] $T$ is the identity operator. Thus $\left(t_{0}\right)^{-1}\left(I-e^{t_{0} N}\right)=0$ on one hand and tends to $N$ as $t_{0} \rightarrow 0$ on the other.

\section{BiBLIOGRAPHY}

1. H. F. Bohenblust and S. Karlin, Geometrical properties of the unit sphere of Banach algebras, Ann. of Math. (2), 62 (1955), 217-229.

2. E. Hille and R. Phillips, Functional analysis and semi-groups, Amer. Math. Soc. Colloq. Publ., vol. 37, Amer. Math. Soc., Providence, R. I., 1957.

3. G. Lumer and R. S. Phillips, Dissipative operators in a Banach space, Pacific J. Math. 11 (1961), 679-698.

University of Minnesota 